\title{
Lipid Polarity and Sorting in Epithelial Cells
}

\author{
Gerrit van Meer and Kai Simons \\ European Molecular Biology Laboratory, D-6900 Heidelberg, Federal Republic of Germany
}

\begin{abstract}
Apical and basolateral membrane domains of epithelial cell plasma membranes possess unique lipid compositions. The tight junction, the structure separating the two domains, forms a diffusion barrier for membrane components and thereby prevents intermixing of the two sets of lipids. The barrier apparently resides in the outer, exoplasmic leaflet of the plasma membrane bilayer. First data are now available on the generation of these differences in Madin-Darby canine kidney (MDCK) cells, grown on filter supports. Experiments in which fluorescent precursors of apical lipids were introduced into the cell have demonstrated that upon biosynthesis apical lipids are sorted from basolateral lipids in an intracellular compartment. In this paper we present a model for the sorting process, the central point of which is that the two sets of lipids laterally segregate into microdomains that bud to form vesicles delivering the lipids to the apical and the basolateral plasma membrane domains, respectively.
\end{abstract}

Key words: lipid phases, sphingolipids, Golgi complex, plasma membrane polarity, membrane domain formation

The plasma membrane of epithelial cells is differentiated into two domains, the apical membrane lining the epithelial lumen and the basolateral domain which faces the blood supply of the tissue [1]. The two plasma membrane domains are responsible for the vectorial functions which characterize transporting, absorptive, and secretory epithelia. The epithelial cells accomplish their polarized functions by localizing a distinct set of cell surface components to either of the two plasma domains. Not only do the apical and the basolateral cell surfaces have distinct protein compositions (enzymes, transport proteins, channels, receptors, etc), but they also contain different lipids. The most complete analysis of the lipid compositions has been reported for intestinal cells $[2-4]$. The major differences in lipid composition are a 2 -4-fold enrichment of glycosphingolipids and a 2-4-fold depletion of phosphatidylcholine (PC) in the apical membrane. The amount of the second major phospholipid, phosphatidylethanolamine (PE), as a percentage of total lipid is about equal in the two domains. In other cell types apical enrichment of the sphingophospholipid, sphingo-

G. van Meer's present address is Department of Cell Biology, Medical Faculty, State University of Utrecht, Nicolaas Beetsstraat 22, 3511 HG Utrecht, The Netherlands.

Received May 19, 1987; accepted June 16, 1987.

(C) 1988 Alan R. Liss, Inc. 
myelin, is also observed [eg, 5,6]. In Madin-Darby canine kidney (MDCK) cells the phospholipid differences between the domains are very similar to those observed in intestinal cells $[7,8]$. MDCK cells also have a very high content of glycosphingolipids, which, as in intestinal cells, are enriched to the apical domain [9-12]. How the polarized distribution of cell surface lipids is generated and maintained in MDCK cells is the subject of this short review.

\section{TIGHT JUNCTIONS MAINTAIN LIPID POLARITY}

The boundary between the two cell surface domains is formed by a specific zone of cell-cell contact, which encircles the top of each cell [13]. This structure connects neighbouring cells and seals the spaces between the cells. The sealing function has been well characterized [14]. In addition, the tight junction has been suggested to have a role in maintaining the compositional differences between the two domains by forming a diffusion barrier in the plane of the membrane [14]. Very little is known about the molecular arrangement at the membrane contact sites of the tight junction. Models for the structure of the tight junction have been devised on the basis of its ultrastructural features [15-17]. At the site of the tight junction the intercellular space is occluded, and the two apposed plasma membranes seem to have fused or merged. In freeze-fracture replicas, a complementary pattern of anastomosing strands and furrows is observed, which reflects the molecular organization of the junctional sites. The peculiarities of the strand structures and the membrane fusion images led Kachar, Pinto da Silva, and Reese to postulate that the strands of the tight junctions represent cylindrical, inverted lipid micelles $[16,17]$. In this lipid model of the zonula occludens, the cytoplasmic leaflets of the apical and the basolateral plasma membrane domains are continuous, while the exoplasmic leaflets are interrupted by the hexagonal lipid cylinder. The model also postulates continuity between the exoplasmic leaflets of the plasma membrane domains of adjacent cells.

Based on the lipid model of the tight junction, interesting predictions concerning the diffusion of membrane lipids can be made. Passage of lipids between the apical and the basolateral domains would depend on their orientation in the bilayer. They would diffuse within the continuous cytoplasmic leaflet from one domain of the cell surface to the other, whereas in the exoplasmic bilayer leaflet the tight junction would act as a barrier between the apical and the basolateral domains. The model further predicts that lipid molecules in the exoplasmic leaflet would be able to diffuse through a continuous lipid monolayer from one cell to another. A study using fluorescent lipid probes indeed suggested that movemert from the apical to the basolateral side took place [18]. However, the use of water-soluble lipids in this study left open the possibility that movement occurred via the cytoplasmic aqueous phase.

We have tried to overcome this problem by a more direct experimental approach to the question of tight junction permeability [8]. First of all, we chose an experimental system that has been carefully studied for basolateral and apical protein polarity: two strains of MDCK cells grown on permeable supports [1]. By growing MDCK cells on filters, conditions are achieved that closely mimic those prevailing in vivo. On filters, the cells feed from the basal side and form a monolayer of cuboidal cells, which is more stable to experimental manipulations than cells grown on solid supports. Second, we developed methods to fuse liposomes with the apical plasma membrane domain $[19,20]$. Because of this we were able to use a fluorescent 
phospholipid, N-rhodamine-dioleoyl PE (N-Rh-PE), that in its water-insolubility is more natural than the analogs previously used. The fusion reaction also made it possible to insert the fluorescent lipid into both leaflets of the plasma membrane bilayer by the use of symmetric liposomes, or primarily into the exoplasmic leaflet by the use of liposomes in which the fluorescent lipid was enriched in the external leaflet. Third, the behavior of the fluorescent lipid was studied at $0^{\circ} \mathrm{C}$, at which temperature diffusion should occur but vesicular transport is completely inhibited. The fusion reaction took place within $1 \mathrm{~min}$ at $37^{\circ} \mathrm{C}$, a time which is too short for transcytosis to play a role in the transport to the basolateral surface [21,22]. Intactness of the tight junction was assessed by measuring the trans-epithelial electrical resistance [23]. Using these methods, we were able to observe clear differences in the behavior of implanted N-Rh-PE in MDCK cells [8]. When fused into the exoplasmic leaflet of the apical plasma membrane, N-Rh-PE did not distribute to the lateral plasma membrane unless the junctions were opened by calcium removal. However, when $\mathrm{N}$ Rh-PE was fused into both the exoplasmic and cytoplasmic leaflets of the bilayer, a fraction of the fluorescent phospholipid immediately passed to the basolateral plasma membrane domain. Also glycolipids have been shown to be unable to pass the tight junction in the exoplasmic bilayer leaflet [24]. These results agree with the predictions of the hexagonal lipid model for tight junction structure.

We also tested whether lipids can diffuse from one epithelial cell to another through continuous exoplasmic leaflets of their apical plasma membranes by studying the behavior of endogenous glycolipids [25]. Strain II MDCK cells, a subline of the MDCK cell line, possess a series of glycolipids, the globo series, which are not found in MDCK strain I cells, a different MDCK subline [10]. Forssman antigen, one glycolipid of this series [GalNAc $(\alpha 1-3) \operatorname{GalNAc}(\beta 1-3) \mathrm{Gal}(\alpha 1-4) \mathrm{Gal}(\beta 1-4) \mathrm{Glc}(\beta 1-$ 1)Cer], constitutes $21 \%$ of the total neutral glycosphingolipids of MDCK strain II cells. When a monolayer of these cells was labeled from the apical side with a monoclonal antibody against Forssman antigen, apical staining was observed [25]. This glycolipid was therefore present in the exoplasmic leaflet of the apical plasma membrane. We co-cultured MDCK strain II cells with MDCK strain I cells which do not express Forssman antigen. The presence of intact tight junctions between the two cell types was demonstrated by adding a monoclonal antibody which recognizes uvomorulin, a protein present on the lateral membrane of MDCK cells [26], and showing that it did not label the lateral membrane unless the tight junctions had been opened by EGTA treatment before fixation. Therefore, tight junctions were present between co-cultured strain I and II cells. Staining with a monoclonal antibody against Forssman antigen demonstrated the presence of antigen on the apical surface of about $50 \%$ of the cells in the mixed monolayer. The boundary between stained and unstained cells was sharp. Thus, the endogenous glycolipid could not pass from MDCK strain II cells to the apical surface of neighboring MDCK strain I cells over a period of 96 hr of coculture at $37^{\circ} \mathrm{C}$.

These observations argue against a continuity between the external leaflets of the apical plasma membrane in adjacent epithelial cells. The results are thus difficult to reconcile with the hexagonal lipid model of tight junction structure where the outer leaflets of the plasma membranes of neighboring cells are fused. It seems more likely that proteins which are part of the tight junction structure [27] bring the plasma membranes of adjacent cells very close together but do not induce a partial fusion. This would also be more consistent with the fact that the tight junction, as a barrier to ion diffusion between cells, displays ion selectivity [14]. 
If one assumes that the free diffusion of lipid molecules in the cytoplasmic leaflet of the plasma membrane leads to an identical lipid composition of the cytoplasmic leaflets of the apical and basolateral plasma membrane domains, interesting consequences arise for the transbilayer distribution of the individual lipid classes in the apical and basolateral membranes. If the exoplasmic leaflet of the apical domain were predominantly occupied by glycosphingolipids, as is probably the case in intestinal cells [see $2-4,8$ ], the phospholipids of the apical domain would be mainly situated in its cytoplasmic leaflet. The phospholipid composition of the cytoplasmic leaflet of the basolateral membrane would be identical with that of the apical domain, and the distribution of the individual phospholipid classes across the basolateral membrane bilayer could be predicted from the total phospholipid composition of the basolateral membrane. For the two major phospholipid classes this leads to the following predictions: $65-90 \%$ of the PE and only $10-25 \%$ of the PC would be localized in the cytoplasmic leaflet $[3,7,8]$. This agrees with the distribution reported for the plasma membrane of erythrocytes and blood platelets [28,29], and may reflect general principles underlying phospholipid organization in mammalian plasma membranes.

\section{SORTING OF PLASMA MEMBRANE LIPIDS IN THE GOLGI COMPLEX}

These observations suggest that the lipid differences between the apical and the basolateral membranes reside in their exoplasmic leaflets. The problem of generating the different lipid compositions of these two membrane domains is reduced to enriching sphingolipids, predominantly glycosphingolipids, and PC in the exoplasmic leaflets of the apical and the basolateral membranes respectively. How are these differences generated? Several possibilities exist. One is local metabolism. This seems very unlikely since PC is synthesized mainly in the endoplasmic reticulum, and glycosphingolipids in the Golgi complex [30,31]. These lipids would therefore have to be transported to the cell surface from their sites of synthesis. In principle, this could occur by two routes, either through the cytosol-eg, by the help of exchange proteins-or by vesicular transport. If vesicular transport were the mechanism for distributing the correct lipids apically and basolaterally, one would expect that sorting would occur in the membrane compartment from which vesicles exit for the cell surface. This is the trans-most compartment of the Golgi complex, the trans-Golgi network [32]. This is also the site where newly synthesized apical and basolateral proteins are thought to be sorted from each other before their transport to the cell surface [33].

In a recent study [34] we addressed the question of whether lipid sorting occurs in the Golgi complex by incorporating into MDCK fluorescent precursor of glycosphingolipids and sphingomyelin, allowing their fluorescent products to accumulate in the membrane of the Golgi complex and monitoring their subsequent delivery to the apical and basolateral cell surface. We used the fluorescent ceramide analog $\mathrm{N}$ 6[7-nitro-2,1,3,-benzoxadiazol-4-yl]amino-caproyl sphingosine (C6-NBD-ceramide) introduced by Lipsky and Pagano [35]. This probe partitions into the membranes of tissue culture cells at $2^{\circ} \mathrm{C}$ and becomes trapped in the Golgi complex upon conversion to C6-NBD-sphingomyelin and C6-NBD-glucosylceramide at $20^{\circ} \mathrm{C}$ (Fig. 1). These products then move to the plasma membrane at $37^{\circ} \mathrm{C}$ from where they can be depleted by means of a "back-exchange" reaction with unlabeled liposomes. The first conclu- 


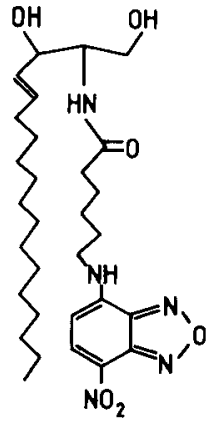

cer

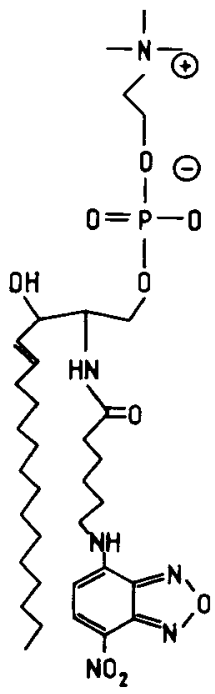

$\mathrm{SPH}$

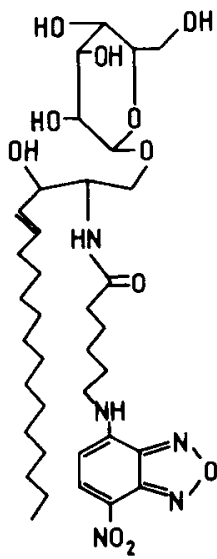

gle-cer

Fig. 1. The C6-NBD ceramide is metabolized to C6-NBD-sphingomyelin and C6-NBD-glucosylceramide in the Golgi complex [35].

sion of our study was that the fluorescent sphingolipids, C6-NBD-sphingomyelin and C6-NBD-glucosylceramide, were selectively incorporated into the luminal leaflet of the membrane of the Golgi complex. Secondly, both fluorescent lipids were delivered to the cell surface by vesicular transport and, most importantly, the cell surface delivery was polar: per unit of surface area, roughly ten times more C6-NBDglucosylceramide and three times more C6-NBD-sphingomyelin were delivered to the apical plasma membrane domain. This enrichment was due to direct delivery to the two surface domains and not a consequence of plasma membrane recycling, since the experiments were performed under conditions in which both domains were continually depleted of newly delivered fluorescent lipid. Sorting must therefore have occurred before the fluorescent lipids reached the plasma membrane.

These results suggest that the cell surface polarity of lipids may be generated by lipid sorting in the trans-Golgi network. The topology of the bilayer differences is compatible with the physical separation of sphingolipids and PC in the luminal leaflet of the Golgi membrane, subsequent budding of the sphingolipid domain into a membrane vesicle, and fusion of the vesicle with the apical membrane introducing the sphingolipids into the exoplasmic leaflet of the apical lipid bilayer (Fig. 2). According to this working hypothesis, the specific event in the lipid sorting process is the generation of the sphingo(glyco)lipid micro-domain in the luminal leaflet of the trans-Golgi network. The luminal leaflet of the membrane which buds into basolateral transport vesicles would, as a consequence, be depleted of the sphingo(glyco)lipids and enriched in PC. This view assumes that the phospholipid asymmetry across the bilayer has been generated earlier in the pathway, possibly already in the membrane of the endoplasmic reticulum [36], and that the asymmetric distribution of choline and amino-containing phospholipids is maintained during the membrane vesiculations 


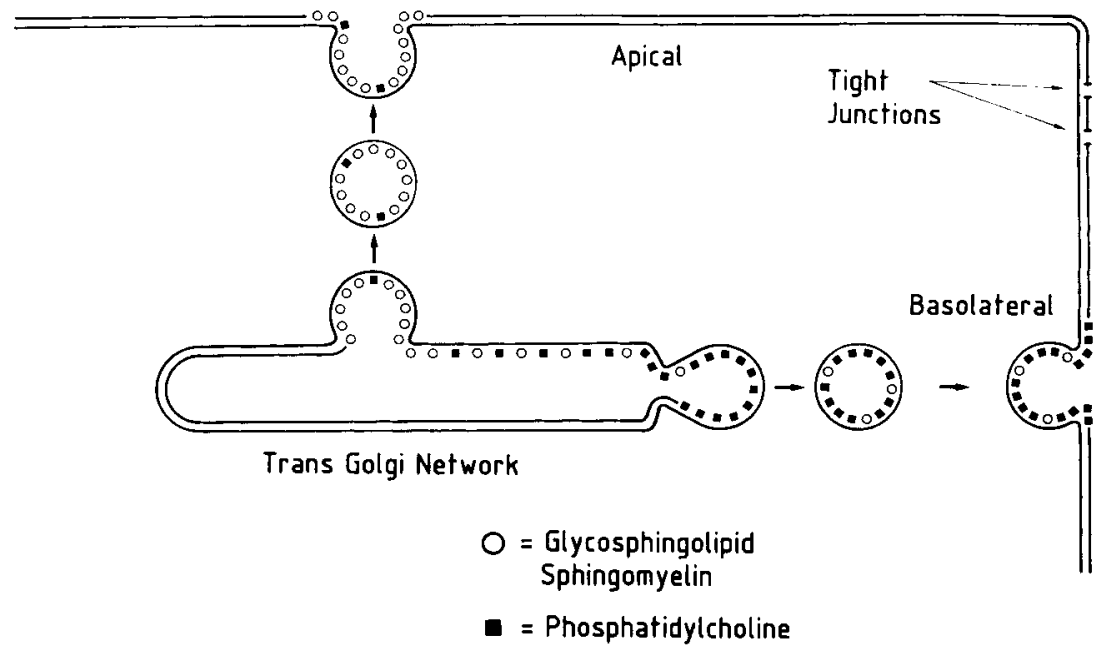

Fig. 2. Sorting of apical and basolateral lipids by lateral segregation in the luminal bilayer leaflet of the trans-Golgi network.

Sphingolipids

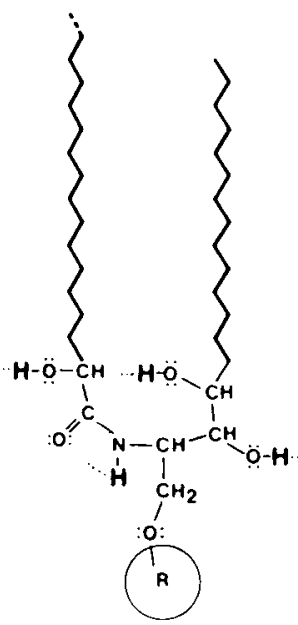

Glycerolipids

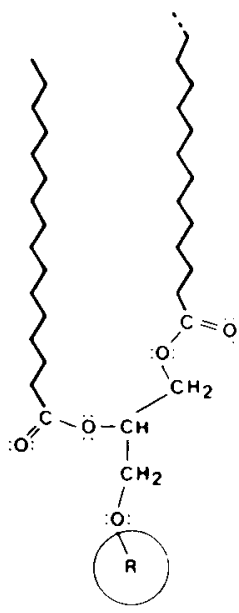

Fig. 3. Differences in hydrogen bond donors and acceptors in the ceramide part of sphingolipids and the diglyceride part of glycerolipids. (Reproduced from Pascher [37] with permission.)

that occur in the biosynthetic transport pathway starting in the endoplasmic reticulum and ending at the cell surface.

Our working hypothesis for lipid sorting in the Golgi complex assumes the formation of a microdomain of sphingo(glyco)lipids in the luminal leaflet of the bilayer. If this were true, this would be the first clear example where the formation of microdomains of lipids is of functional importance for the cell. One interesting feature of the process is that the sphingolipids have a common structural feature, their sphingosine backbone which is not present in glycerolipids. These sphingolipids, especially the glycosphingolipids, have a tendency to associate by hydrogen bond 
formation to form separate domains in model lipid systems [37,38]. This domain formation could be facilitated by the conditions prevailing in the lumen of the intracellular sorting compartment (low $\mathrm{pH}$, ionic conditions, etc.). Glycerolipids cannot form intermolecular hydrogen bonds in the same regions of the lipid bilayer since they only have groups which can act as hydrogen bond acceptors (Fig. 3).

Until the putative transport vesicles carrying sphingolipids to the apical membrane have been isolated, we will not know whether these also are responsible for transporting apical proteins to their destination. The delivery of apical lipids and proteins show the same kinetics, and are both slowed down by the $20^{\circ} \mathrm{C}$ temperature block. It is thus hard to imagine that the two sorting processes would not be related. The enticing possibility exists that specific sorting in membrane traffic involves both proteins and lipids interacting with each other in a cooperative fashion to form a lattice which buds into a vesicle. The sphingolipids could bind to sorting proteins and/or apical proteins during microdomain formation in the trans-Golgi network. This interaction would have to be reversed upon arrival at the apical surface domain (change of $\mathrm{pH}$, ionic conditions, etc) to allow the putative sorting proteins to return to the trans-Golgi network to repeat their sorting functions.

\section{ACKNOWLEDGMENTS}

We are grateful to Thomas Gabran and Hilkka Virta for the excellent technical assistance in the experimental work reviewed here, and to Mark Bennett for a critical reading of the manuscript.

\section{REFERENCES}

1. Simons K, Fuller SD: Annu Rev Cell Biol 1:243-288, 1985.

2. Forstner GG, Wherrett JR: Biochim Biophys Acta 306:446-459, 1973.

3. Kawai K, Fujita M, Nakao M: Biochim Biophys Acta 369:222-233, 1974

4. Brasitus TA, Schachter D: Biochemistry 19:2763-2769, 1980.

5. Meier PJ, Sztul ES, Reuben A, Boyer JL: J Cell Biol 98:991-1000, 1984.

6. Molitoris BA, Simon FR: J Membr Biol 83:207-215, 1985.

7. van Meer G, Simons K: EMBO J 1:847-852, 1982.

8. van Meer G, Simons K: EMBO J 5:1455-1464, 1986.

9. Hougland AE, Gaush C, Mavberry WR: Biochem J 177:311-317, 1979.

10. Hansson GC, Simons K, van Meer G: EMBO J 5:483-489, 1986.

11. Nichols GE, Lovejoy JC, Borgman CA, Sanders JM, Young Jr, WW: Biochim Biophys Acta 887:1$12,1986$.

12. Nimura Y, Ishizuka I: J Biochem (Tokyo) 100:825-835, 1986.

13. Farquhar MG, Palade GE: J Cell Biol 17:375-412, 1963.

14. Diamond JM: Physiologist 20:10-18, 1977.

15. Hirokawa N: J Ultrastruct Res 80:288-301, 1982.

16. Pinto da Silva P, Kachar B: Cell 28:441-450, 1982.

17. Kachar B, Reese TS: Nature 296:464-466, 1982.

18. Dragsten PR, Blumenthal R, Handler JS: Nature 294:718-722, 1981.

19. van Meer G, Simons K: J Cell Biol 97:1365-1374, 1983.

20. van Meer G, Davoust J, Simons K: Biochemistry 24:3593-3602, 1985.

21. Pesonen M, Ansorge W, Simons K: J Cell Biol 99:796-802, 1984.

22. von Bonsdorff C-H, Fuller SD, Simons K: EMBO J 4:2781-2792, 1985.

23. Cereijido M, Robbins ES, Dolan WJ, Rotunno CA, Sabatini DD: J Cell Biol 77:853-880, 1978.

24. Spiegel S, Blumenthal R, Fishman PH, Handler JS: Biochim Biophys Acta 821:310-318, 1985.

25. van Meer G, Gumbiner B, Simons K: Nature 322:639-641, 1986.

26. Gumbiner B, Simons K: J Cell Biol 102:457-468, 1986.

27. Stevenson BR, Siliciano JD, Mooseker MS, Goodenough DA: J Cell Biol 103:755-766, 1986.

28. Bretscher MS: J Mol Biol 71:523-528, 1972.

29. Op den Kamp JAF: Annu Rev Biochem 48:47-71, 1979.

30. Bell RM, Ballas LM, Coleman RA: J Lipid Res 22:391-403, 1981. 
31. Pagano RE, Sleight RG: Trends Biochem Sci 10:421-425, 1985.

32. Griffiths G, Simons K: Science 234:438-443, 1986.

33. Hughson E, Wandinger-Ness A, Gausepohl H, Griffiths G, Simons K: In Schwartz M (ed): "The Centenary Symposium of the Pasteur Institute", (in press).

34. van Meer G, Stelzer EHK, Wijnaendts van Resandt RW, Simons K: J Cell Biol 105:1623-1635, 1987.

35. Lipsky NG, Pagano RE: Science 228:745-747, 1985.

36. Bishop WR, Bell RM: Cell 42:51-60, 1985.

37. Pascher I: Biochim Biophys Acta 455:433-451, 1976.

38. Thompson TE, Barenholz Y, Brown RE, Correa-Freire M, Young WW, Tillack TW: In Freysz L (ed): "Enzymes of Lipid Metabolism." New York: Plenum Press, 1986, pp 387-396. 